\section{Oxidative Stress, Endogenous Antioxidant and \\ Ischemia-modified Albumin in Normolipidemic Acute Myocardial Infarction Patients}

\author{
Arun Kumar, ${ }^{, a}$ Ramiah Sivakanesan, ${ }^{b}$ \\ and Sandeep Singh ${ }^{a}$
}

${ }^{a}$ Department of Biochemistry, Hindustan Institute of Medical Sciences and Research, Knowledge Park-III Plot No: 32, 34, Greater Noida, Uttar Pradesh, India and ${ }^{b}$ Department of Biochemistry, Faculty of Medicine, University of Peradeniya, Kandy, Sri Lanka 20400

(Received March 3, 2008; Accepted May 15, 2008)

Dyslipidemia appears to be a conventional risk factor for acute myocardial infarction (AMI). Several studies have reported decreased levels of antioxidants and increased levels of ischemia-modified albumin (IscMA) and lipid peroxides in dyslipidemic myocardial infarct patients. However, literature search reveals no reports of normolipidemic AMI patients with reference to antioxidants and IscMA studies. Therefore, this study determined the endogenous levels of antioxidants and IscMA in normolipidemic AMI patients so that prospective measures could be taken to avoid acute coronary complications. The serum lipid profile, albumin, uric acid, total bilirubin, malondialdehyde, conjugated dienes and IscMA levels were determined in 165 normolipidemic AMI patients and 165 age/sex-matched controls. In addition, serum lipid concentrations were estimated by enzymatic methods. Endogenous antioxidants were significantly decreased in AMI patients compared with controls. In parallel with this, serum malondialdehyde (MDA) and conjugated dienes were significantly increased in AMI patients compared with controls. IscMA levels were significantly increased in AMI patients compared with controls. As for the serum lipid profile, total cholesterol (TC), cholesterol in low density lipoprotein (LDL) and triglycerides (TG) were higher in AMI subjects. High-density lipoprotein (HDL)-to-cholesterol and LDL-cholesterol-to-HDLcholesterol ratios were also greater in AMI subjects.

\footnotetext{
*To whom correspondence should be addressed: Department of Biochemistry, Hindustan Institute of Medical Science and Research, Knowledge Park-III Plot No: 32, 34, Greater Noida, Uttar Pradesh, India. Tel.: +91-1203212361; Fax: +911202323617; E-mail: arun732003@gmail.com
}

However, HDL-cholesterol was lower in AMI patients than control. AMI is a multifactorial disease that can arise even in normolipidemic subjects. The present study suggests that measuring of serum antioxidants and IscMA in normolipidemic patients would provide an index of oxidative stress and ischemia due to structural modifications of circulating albumin in serum.

Key words — acute myocardial infarction, normal lipid profile, endogenous antioxidant, ischemia-modified albumin

\section{INTRODUCTION}

With the explosive rise in the incidence of coronary artery disease (CAD), this entity is predicted to be the leading cause of morbidity and mortality in developing countries by the year 2015. ${ }^{1)}$ It is a multifactorial disease and predisposing factors suggested so far are hereditary, hyperlipidemia, obesity, hypertension, and environmental factors and life style variables such as stress, smoking and alcohol consumption. ${ }^{2)}$ Lipoprotein profile is found to be deranged in the large proportion of CAD patients, especially Asians showing a mixed picture of dyslipidemia. Dyslipidemia is a well-established risk factor as reported in many studies. ${ }^{3-10)}$ Lowering of high-density lipoprotein (HDL)-cholesterol (HDL-C) is a common phenomenon observed in myocardial infarction (MI) patients. ${ }^{3-10)}$ Elevated Low density lipoprotein (LDL)-cholesterol (LDLC) is considered the most important risk factor for CAD and its oxidation plays a central role in atherogenesis. Subendothelial accumulation of foam cells initiates the process of atherosclerosis. The generation of foam cells depends on the uptake of oxidized LDL by macrophages via scavenger receptors, leading to accumulation of fatty streaks and more complex fibro fatty or atheromatous plaques. 11) Under oxidative stress, not only LDL but also other serum lipids are exposed to oxidation. Free radicals play an important role in the pathogenesis of tissue damage in many different clinical disorders. ${ }^{12)}$ Oxygen free radicals (OFRs) are produced continuously. Normally, OFR is regulated by a balance between tissue oxidant and antioxidant activity. ${ }^{13)}$ The latter is achieved by the antioxidant scavenger system which includes enzymes (superoxide dismutase, catalase and glutathione peroxidase) and antioxidant vitamins $\mathrm{C}, \mathrm{A}$, and $\mathrm{E}$ and $\alpha$-lipoic acid 
and other carotenoids. ${ }^{14)}$ The endogenous antioxidant system includes albumin, uric acid, and total bilirubin. Imbalance of this reaction either due to excess free radical formation or insufficient removal by antioxidants leads to oxidative stress. ${ }^{15)}$ Most cases of ischemia cause a reduction in oxygen supply to the cells but do not cause cell death. Ideally, one should be able to identify myocardial ischemia before it progresses to irreparable myocardial cell damage. Most of the cardiac markers do not become positive until cell death occurs and provide reliable information when measured in the first $2-6 \mathrm{hr}$ following ischemic events. ${ }^{16)}$

Recently, a new parameter, ischemia-modified albumin (IscMA), has been developed and found very useful for the detection of acute myocardial ischemia. While the circulating albumin molecules in the presence of ischemia are modified, this modification does not trigger cell death. ${ }^{17)}$ However, literature survey reveals that dyslipidemic patients are more prone to MI due to increased free radical generation and ischemic process causing enhanced formation of IscMA. Several studies have reported an increase in IscMA and lipid peroxidation concomitant with lowering of antioxidants. ${ }^{18)}$ It has been also reported that IscMA is elevated in acute MI (AMI) patients with dyslipidemia. ${ }^{19,20)}$ In our hospital, we treat a number of normolipidemic myocardial infarct patients. Hence the present study was undertaken to evaluate the levels of IscMA as well as antioxidants and lipid peroxides in normolipidemic AMI patients, as no such study has been conducted so far.

\section{MATERIALS AND METHODS}

Setting Design and Patients — The study consisted of 165 patients (123 men and 42 women) with AMI, admitted to the Intensive Cardiac Care Unit, Sharda Hospital, India. The diagnosis of AMI was established according to diagnostic criteria: chest pain lasting for $\leq 3 \mathrm{hr}$, electrocardiographic (ECG) changes (ST elevation $\geq 2 \mathrm{~mm}$ in at least two leads) and elevation in enzymatic activities of serum creatine phosphokinase and aspartate aminotransferase. The control group consisted of 165 age/sex-matched healthy volunteers (123 men and 42 women). The design of this study was pre-approved by the institutional ethical committee board of Chaudhary Charan Singh University, and informed consent was obtained from the patients and controls. Inclusion cri- teria were patients with a diagnosis of AMI with normal lipid profile. Patients with diabetes mellitus, renal insufficiency, current and past smokers, hepatic disease or taking lipid lowering drugs or antioxidant vitamin supplements were excluded from the study. Normolipidemic status was judged by the following criteria: LDL $\leq 160 \mathrm{mg} / \mathrm{dl}$; HDL, $\geq 35 \mathrm{mg} / \mathrm{dl}$; total cholesterol (TC), $<200 \mathrm{mg} / \mathrm{dl}$; and triglycerides (TG), $<150 \mathrm{mg} / \mathrm{dl} .^{21)}$ Ten milliliters of blood was collected after overnight fasting for lipid profile assay. For IscMA analysis, $2 \mathrm{ml}$ of blood was collected from the patients immediately after admission to intensive care unit.

Lipid Profile — TC, TG and HDL-C were analyzed enzymatically using kit obtained from Randox Laboratories Limited, Crumlin, U.K. Plasma LDL-C was determined from the values of TC and HDL-C using the following formula:

$$
\text { LDL-C }=\text { TC }-\frac{\text { TG }}{5}-\text { HDL-C }(\mathrm{mg} / \mathrm{dl})
$$

Serum Albumin — Serum albumin was measured by Bromocresol green binding method. ${ }^{22)}$

Other Assays — All chemicals of analytical grade were obtained from Sigma-Aldrich Company, New Delhi, India. Serum uric acid was estimated by the method of Brown based on the development of a blue color due to tungsten blue as phosphotungstic acid is reduced by uric acid in alkaline medium. ${ }^{23)}$ Serum total bilirubin was estimated by the method of Jendrassik and Grof. ${ }^{24)}$ Malondialdehyde (MDA) derived from lipid peroxides was determined as a thiobarbituric acid (TBA)-reactive substance. ${ }^{25)}$ Conjugated dienes (CD) were measured according to the method of Recknagel and Glende. ${ }^{26)}$

IscMA concentration was determined by addition of a known amount of cobalt(II) to a serum sample and measurement of the unbound cobalt(II) by the intensity of colored complex formed after reacting with dithiothreitol (DTT) by colorimeter. ${ }^{27)}$ An inverse relationship thus exists between the level of albumin bound cobalt and the intensity of the color formed. The preparations for the Co(II) albumin binding protocol involved the addition of $200 \mu \mathrm{l}$ of patient serum to $50 \mu \mathrm{l}$ of a solution of $1 \mathrm{~g} / \mathrm{l}$ cobalt chloride, followed by vigorous mixing and $10-\mathrm{min}$ incubation. Dithiothreitol $(50 \mu \mathrm{l}$ of a $1.5 \mathrm{~g} / \mathrm{l}$ solution) was then added and mixed. After 2-min incubation, $1.0 \mathrm{ml}$ of a $9.0 \mathrm{~g} / \mathrm{l}$ solution of $\mathrm{NaCl}$ was added. The absorbance of the assay mixture was read at $470 \mathrm{~nm}$. The blank was prepared similarly 
with the exclusion of DTT. The values are expressed in U/ml. IMA assay was standardized in the Department of Biochemistry and a standard curve was prepared in the range $6.0-60.0 \mu \mathrm{g} \mathrm{CoCl}_{2} / \mathrm{ml}$. One IMA unit was defined as " $\mu \mathrm{g}$ of free $\mathrm{Co}(\mathrm{II})$ in the reaction mixture per ml of serum sample."

Statistical Analysis — The data from patients and controls were compared by Student's $t$-test. Values are expressed as mean \pm standard deviation (S.D.). Microsoft Excel for Windows 2000 was used for statistical analysis. $p$-value $<0.05$ was considered to indicate statistical significance.

\section{RESULTS}

Serum parameters in AMI patients and control are shown in Table 1, and the data in males and females are separately shown in Tables 2 and 3, respectively. TC, its ratio to HDL-C (TC/HDL-C) and TG were significantly higher in both sexes of AMI subjects compared with control (Tables 1-3). Significant difference for HDL-C between AMI and control was seen only in female subjects (Table 3). On the other hand, LDL-C and its ratio to HDL-C (LDL-C/HDL-C) were higher in male AMI subjects than in control (Table 2). The status of endogenous antioxidants (albumin, bilirubin and uric acid) and lipid peroxidation (MDA and CD) are shown in Ta-

Table 1. Endogenous Antioxidants, Lipid Peroxidation, Lipid Profile and Ischemia-modified Albumin in AMI Patients and Healthy Controls (mean \pm S.D.)

\begin{tabular}{lcccc}
\hline \hline Variables & Controls $(n=165)$ & Patients $(n=165)$ & $p$-value $(95 \% \mathrm{CI})$ \\
\hline Age & $60.6 \pm 4.0$ & $61.8 \pm 4.0$ & $0.004(61.26-62.42)$ \\
Total Cholesterol (TC) mg\% & $168.6 \pm 12.2$ & $186.4 \pm 14.0$ & $<0.001(184.31-188.56)$ \\
HDL-Cholesterol (HDL-C) $\mathrm{mg} \%$ & $50.5 \pm 6.8$ & $41.3 \pm 4.6$ & $<0.001(40.56-41.97)$ \\
TC/ HDL-C ratio & $3.4 \pm 0.4$ & $4.6 \pm 0.6$ & $<0.001(4.48-4.65)$ \\
Triglycerides (TG) mg\% & $107.8 \pm 11.5$ & $129.0 \pm 12.2$ & $<0.001(127.10-130.82)$ \\
LDL-Cholesterol (LDL-C) mg\% & $83.6 \pm 12.0$ & $119.4 \pm 14.1$ & $<0.001(17.22-21.51)$ \\
LDL-C/HDL-C ratio & $1.9 \pm 0.3$ & $2.9 \pm 0.5$ & $<0.001(2.85-3.00)$ \\
TG/HDL-C ratio & $2.2 \pm 0.4$ & $3.2 \pm 0.5$ & $0.3149(3.086-3.234)$ \\
Albumin mg\% & $4.4 \pm 0.3$ & $4.2 \pm 0.3$ & $<0.001(4.17-4.28)$ \\
Total Bilirubin mg\% & $0.8 \pm 0.2$ & $0.7 \pm 0.2$ & $<0.001(0.62-0.69)$ \\
Uric acid mg\% & $5.8 \pm 1.3$ & $4.3 \pm 1.0$ & $<0.01(4.18-4.45)$ \\
MDA (nmol/l) & $5.7 \pm 1.0$ & $14.8 \pm 1.7$ & $<0.02(11.55-15.06)$ \\
Conjugated dienes $(\mu \mathrm{mmol} / \mathrm{l})$ & $31.0 \pm 2.7$ & $48.3 \pm 5.5$ & $<0.001(47.44-49.11)$ \\
Ischemia modified albumin $(\mathrm{U} / \mathrm{ml})$ & $81.9 \pm 3.9$ & $97.5 \pm 11.7$ & $<0.001(95.71-99.28)$ \\
\hline
\end{tabular}

Table 2. Endogenous Antioxidants, Lipid Peroxidation, Lipid Profile and Ischemia-modified Albumin in Male Patients and Healthy Controls (mean \pm S.D.)

\begin{tabular}{|c|c|c|c|}
\hline Variables & Control Male $(n=123)$ & Male Patients $(n=123)$ & $p$-value $(95 \% \mathrm{CI})$ \\
\hline Age & $60.7 \pm 4.1$ & $61.5 \pm 3.3$ & $0.034 \quad(60.95-62.10)$ \\
\hline Total Cholesterol (TC) $\mathrm{mg} \%$ & $168.1 \pm 12.1$ & $183.8 \pm 13.7$ & $<0.001 \quad(182.41-186.25)$ \\
\hline HDL-Cholesterol (HDL-C) mg\% & $49.9 \pm 7.3$ & $41.8 \pm 4.9$ & $0.0801(40.91-42.64)$ \\
\hline TC/ HDL-C ratio & $3.4 \pm 0.3$ & $4.5 \pm 0.6$ & $<0.001 \quad(\quad 4.34-4.55)$ \\
\hline Triglycerides (TG) $\mathrm{mg} \%$ & $105.0 \pm 10.3$ & $126.2 \pm 11.7$ & $<0.001 \quad(124.14-128.29)$ \\
\hline LDL-Cholesterol (LDL-C) $\mathrm{mg} \%$ & $80.0 \pm 8.0$ & $116.8 \pm 13.8$ & $<0.001 \quad(114.38-119.25)$ \\
\hline LDL-C/HDL-C ratio & $1.9 \pm 0.3$ & $2.8 \pm 0.5$ & $<0.001 \quad(\quad 2.74-\quad 2.93)$ \\
\hline TG/HDL-C ratio & $2.2 \pm 0.4$ & $3.1 \pm 0.5$ & $0.0123(2.97-3.14)$ \\
\hline Albumin mg\% & $4.5 \pm 0.3$ & $4.2 \pm 0.4$ & $<0.001 \quad(\quad 4.14-4.27)$ \\
\hline Total Bilirubin mg\% & $0.8 \pm 0.2$ & $0.7 \pm 0.2$ & $<0.001 \quad(\quad 0.65-\quad 0.72)$ \\
\hline Uric acid mg\% & $5.4 \pm 1.1$ & $4.3 \pm 0.9$ & $<0.001 \quad(\quad 4.18-4.49)$ \\
\hline MDA (nmol/l) & $5.7 \pm 1.0$ & $14.8 \pm 1.7$ & $<0.001 \quad(14.44-15.07)$ \\
\hline Conjugated dienes $(\mu \mathrm{mol} / \mathrm{l})$ & $31.0 \pm 2.7$ & $48.3 \pm 5.5$ & $<0.001 \quad(45.88-47.77)$ \\
\hline Ischemia modified albumin (U/ml) & $81.7 \pm 3.9$ & $97.9 \pm 12.2$ & $<0.001 \quad(95.71-100.02)$ \\
\hline
\end{tabular}


Table 3. Endogenous Antioxidants, Lipid Peroxidation, Lipid Profile and Ischemia-modified Albumin in Female Patients and Healthy Controls (mean \pm S.D.)

\begin{tabular}{|c|c|c|c|}
\hline Variables & Control Female $(n=42)$ & Patients Female $(n=42)$ & $p$-value $(95 \% \mathrm{CI})$ \\
\hline Age & $60.5 \pm 2.9$ & $62.7 \pm 5.0$ & $0.036 \quad(61.22-64.23)$ \\
\hline Total Cholesterol (TC) $\mathrm{mg} \%$ & $170.0 \pm 12.4$ & $194.0 \pm 13.0$ & $<0.001 \quad(190.08-197.97)$ \\
\hline HDL-Cholesterol (HDL-C) mg\% & $52.3 \pm 4.6$ & $39.8 \pm 3.4$ & $<0.001 \quad(38.75-40.78)$ \\
\hline TC/HDL-C ratio & $3.3 \pm 0.5$ & $5.0 \pm 0.4$ & $<0.001 \quad(\quad 4.82-\quad 5.09)$ \\
\hline Triglycerides (TG) $\mathrm{mg} \%$ & $116.1 \pm 11.0$ & $137.0 \pm 9.8$ & $<0.001 \quad(134.02-139.95)$ \\
\hline LDL-Cholesterol (LDL-C) mg\% & $94.5 \pm 14.8$ & $126.9 \pm 12.2$ & $0.2044(123.16-130.55)$ \\
\hline LDL-C/HDL-C ratio & $1.8 \pm 0.4$ & $3.2 \pm 0.4$ & $0.3066(3.08-3.33)$ \\
\hline TG/ HDL-C ratio & $2.2 \pm 0.3$ & $3.5 \pm 0.4$ & $<0.001 \quad(\quad 3.34-\quad 3.59)$ \\
\hline Albumin mg\% & $4.4 \pm 0.3$ & $3.6 \pm 0.2$ & $<0.001 \quad(3.53-\quad 3.66)$ \\
\hline Total Bilirubin $\mathrm{mg} \%$ & $0.7 \pm 0.2$ & $0.6 \pm 0.1$ & $<0.001 \quad(\quad 0.53-0.62)$ \\
\hline Uric acid mg\% & $5.7 \pm 1.4$ & $4.3 \pm 1.0$ & $<0.001 \quad(3.98-4.53)$ \\
\hline MDA (nmol/l) & $5.7 \pm 1.0$ & $14.8 \pm 1.7$ & $<0.001 \quad(14.61-15.30)$ \\
\hline Conjugated dienes $(\mu \mathrm{mol} / \mathrm{l})$ & $31.0 \pm 2.7$ & $48.3 \pm 5.5$ & $<0.01 \quad(51.36-53.31)$ \\
\hline Ischemia modified albumin (U/ml) & $82.4 \pm 4.1$ & $96.4 \pm 10.2$ & $<0.001 \quad(93.33-99.48)$ \\
\hline
\end{tabular}

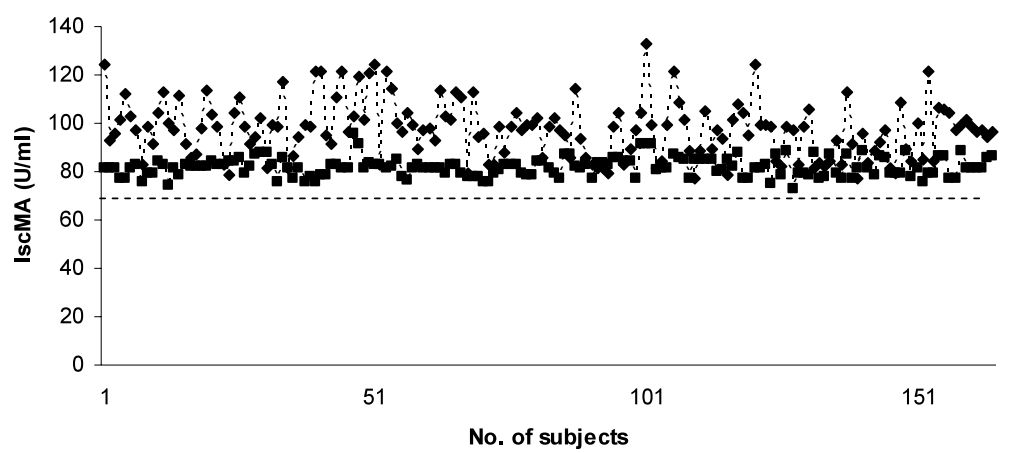

Fig. 1. IscMA in AMI ( $\bullet$ ) and Control Subjects ( $\bullet)$

bles 1-3. All endogenous antioxidants were significantly decreased in AMI patients compared with controls. In agreement with this serum MDA and $\mathrm{CD}$ were more abundant in AMI patients compared with controls. IscMA levels were also greater in both male and female AMI patients compared with control (Tables 1-3). The levels of IscMA in AMI and control subjects are depicted in form of graph (Fig. 1).

\section{DISCUSSION}

Atherosclerosis is one of the major causes of AMI. Contrary to earlier belief, research in the last two decades has shown that atherosclerosis is neither a degenerative disease nor inevitable due to ageing. Atherosclerosis seems to be a chronic inflammatory condition that can be converted to an acute clinical event by the induction of plaque rup- ture, which in turn leads to thrombosis. Hence inflammation occurs during all phases of atherosclerosis, although it must smolder for decades before resulting in a clinical event such as AMI. ${ }^{27)} \mathrm{My}$ ocardial ischemia is caused due to increased oxygen demand exceeding its supply and, if this condition is not reversed, MI precipitates. During this phase, circulating albumin becomes modified at the N-terminal residues, thus decreasing its affinity to bind to $\mathrm{Co}(\mathrm{II})$. Reperfusion of ischemic myocardium not only restores the blood supply but also causes massive production of free radicals, resulting in imbalance between oxidative and anti-oxidative processes. Excess production of reactive oxygen species may initiate lipid peroxidation in cell membranes. These processes reduce the contractile function of the heart and lead to severe myocardial cell damage termed as reperfusion injury. ${ }^{28)}$ This study suggests that antioxidants depletion has a significant impact on the precipitation of MI and these findings 
are consistent with the notion that elevated levels of antioxidants are protective against AMI. ${ }^{29)}$

The current study observed a significant reduction in albumin, uric acid and total bilirubin and a significant increase in MDA, conjugated dienes and IscMA in AMI patients. The findings of the present study are similar to those of the study conducted by Dubois Rande et al. ${ }^{30)}$ and Mc Murray ${ }^{31)}$ which reported a significant rise in MDA level and lipid peroxidation, with a concomitant decrease in antioxidants in patients with unstable angina and chronic heart failure. The present study also concurs with studies of Verma et al., ${ }^{32)}$ who demonstrated that there was a significant drop in antioxidant, whereas lipid peroxides were significantly higher in AMI patients, compared with controls. The findings of the present study are also in good agreement with those of the study conducted by Kharb ${ }^{33)}$ where significantly decreased levels of antioxidants and increased levels of lipid peroxides were reported. This indicates that the antioxidant system combating oxidative stress and inflammation is severely impaired in AMI patients. The findings of the present study indicate that the existence of an abnormal balance between the oxidative and protective mechanisms in patients can be a causative factor for the occurrence of AMI. As has been demonstrated from reperfusion study in ischemic myocardium, ${ }^{31)}$ increased MDA levels in plasma have been used as an index of free radical-mediated damage, which is significantly elevated in AMI patients.

That the IscMA level in AMI patients was significantly higher than in controls as observed in the present study is similar to previous reports. ${ }^{18,20,34)}$ Even though determination of IscMA is promising for the prediction of AMI, its use should be limited until further studies reveal its validity as a biomarker for AMI and usefulness in constructing treatment plans in acute coronary syndrome patients. An extensive study with increased number of patients would be required to compare IscMA with other markers such as troponins and myoglobin. Whether IscMA can be an additional parameter along with troponins to boost the confidence of clinicians in ruling out cardiac ischemia would be of particular interest.

In conclusion, MI is a multifactorial disease that can arise even in normolipidemic subjects. Hence, earlier concepts of maintaining lipid profile within normal limits to prevent MI may be overruled. The present study suggests that measuring serum antioxidants and IscMA in normolipidemic patients will aid better prognosis and management of patients with acute coronary syndromes. Oxidative stress appears an etiological factor for MI as a consequence of free radical scavengers namely antioxidants which tends to be lower in AMI patients.

\section{REFERENCES}

1) Reddy, K. S. (1993) Cardiovascular disease in India. World Health Stat. Q, 46, 101-107.

2) Chopra, V. and Wasir, H. (1998) Implications of lipoprotein abnormalities in Indian patients. $J$. Assoc. Physicians India, 46, 814-818.

3) Mishra, T. K., Routray, S. N., Patnaik, U. K., Padhi, P. K., Satapathy, C. and Behera, M. (2001) Lipoprotein (a) and Lipid Profile in Young Patients with Angiographically Proven Coronary Artery Disease. Indian Heart J., 53, 565-590.

4) Malhotra, P., Kumari, S., Singh, S. and Varma, S. (2003) Isolated Lipid Abnormalities in Rural and Urban Normotensive and Hypertensive North-West Indians. J. Assoc. Physicians India, 51, 459-463.

5) Achari, V. and Thakur, A. K. (2004) Association of Major Modifiable Risk factors Among Patients with Coronary Artery Disease-A retrospective Analysis. J. Assoc. Physicians India., 52, 103-108.

6) Mishra, A., Luthra, K. and Vikram, N. K. (2005) Dyslipidemia in Asian Indians: Determinants and Significance. J. Assoc. Physicians India., 52, 137142.

7) Rajasekhar, D., Srinivasa Rao, P. V., Latheef, S. A., Saibaba, K. S. and Subramanyam, G. (2004) Association of serum antioxidants and risk of coronary heart disease in South Indian population. Indian. J. Med. Sci., 58, 465-471.

8) Rani, S. H., Madhavi, G., Ramachandra Rao, V., Sahay, B. K. and Jyothy, A. (2005) Risk factors for coronary heart disease in type II diabetes. Indian $\mathrm{J}$. Clin. Biochem., 20, 75-80.

9) Ghosh, J., Mishra, T. K., Rao, Y. N. and Aggarwal, S. K. (2006) Oxidised LDL, HDL Cholesterol, LDL Cholesterol levels in patients of Coronary Artery Disease. Indian J. Clin. Biochem., 21, 181-184.

10) Patil, N., Chavan, V. and Karnik, N. D. (2007) Antioxidant Status in Patients with Acute Myocardial Infarction. Indian J. Clin. Biochem., 22, 45-51.

11) Paul, H., Johan, V., Stefaan, J., Frans, Van de W. and Désiré, C. (1998) Oxidized LDL and Malondialdehyde-Modified LDL in Patients with Acute Coronary Syndromes and Stable Coronary Artery Disease. Circulation, 98, 1487-1494.

12) Halliwell, B., Gutteridge, G. M. C. and Cross, C. 
E. (1992) Free radicals and antioxidants and human disease: where we are now? J. Lab. Clin. Invest., 119, 589-620.

13) Frei, B., Stocker, R. and Ames, B. N. (1988) Antioxidant defenses and lipid peroxidation in human blood plasma. Proc. Natl. Acad. Sci. U.S.A., 85, 9748-9752.

14) Shrinivas, K., Vijaya Bhaskar, M., Aruna Kumari, M., Nagaraj, K. and Reddy, K. K. (2000) Antioxidants, lipid peroxidation and lipoproteins in primary hypertension. Indian Heart J., 52, 285-288.

15) Maritim, A. C., Sanders, R. A. and Watkins, J. B. (2003) Diabetes, oxidative stress, and antioxidants: a review. J. Biochem. Mol. Toxicol., 17, 24-38.

16) Gomez, M. A., Anderson, J. L., Karagounis, L. A., Muhlestein, J. B. and Mooers, F. B. (1996) An emergency medicine based protocol for rapidly ruling out myocardial ischemia reduces hospital time and expense. Results of randomized study (ROMO). J. Am. Coll. Cardiol., 28, 25-33.

17) Christenson, R. L., Duh, S. H., Sanhai, W. R., Wu, W. A. B., Holtman, V., Painter, P., Branham, E., Apple, F. S., Murakami, M. A. and Morris, D. L. (2001) Characteristics of an Albumin Cobalt Binding Test for Assessment of Acute Coronary Syndrome Patients: A Multicenter Study. Clin. Chem., 47, 464470.

18) Sinha, M. K., Roy, D., Gaze, D. C. Collinson, P. O. and Kaski, J. C. (2004) "Role of Ischemia Modified Albumin" a new biochemical marker of myocardial Ischaemia, in the early diagnosis of acute coronary syndromes. Emerg. Med. J., 21, 29-34.

19) Robert, H. C., Show, H. D., Wendy, R. S., Alan, H. B. W., Verena, H., Pennell, P., Elizabeth, B., Fred, S. A., MaryAnn, M. and Deborah, L. M. (2001) Characteristics of an Albumin Cobalt Binding Test for Assessment of Acute Coronary Syndrome Patients: A Multicenter Study. Clin. Chem., 47, 464-470.

20) Collinson, P. O., Gaze, D. C., Bainbridge, K., Morris, F., Morris, B., Price, A. and Goodacre, S. (2006) Utility of admission cardiac troponin and "Ischemia Modified Albumin" measurements for rapid evaluation and rule out of suspected acute myocardial infarction in the emergency department. Emerg. Med. J., 23, 256-261.

21) Executive Summary of The Third Report of The National Cholesterol Education Program (NCEP) Expert Panel on Detection, Evaluation, and Treatment of High Blood Cholesterol in Adults (Adult
Treatment Panel III) (2001) Expert Panel of Detection, Evaluation, and Treatment of High Blood Cholesterol in Adults. JAMA, 285, 2486-2497.

22) Perry, B. W. and Doumas, B. T. (1979) Effect of heparin on albumin determination by use of bromocresol green and bromocresol purple. Clin. Chem., 25, 1520-1522.

23) Brown, H. (1945) The determination of uric acid in human blood. J. Biol. Chem., 158, 601-608.

24) Jendrassik, J. and Grof, P. (1938) Vereinfachte photometrische Methoden Zur Beetimmung des Blutbilirubin. Biochem. Z., 297, 81-89.

25) Bernheim, S., Bernheim, M. L. C. and Wilbur, K. M. (1948) The reaction between thiobarbituric acid and the oxidant product of certain lipids. J. Biol. Chem., 174, 257-264.

26) Recknagel, R. O. and Glende, E. A. (1984) Spectrophotometric detection of lipid conjugated dienes. Methods Enzymol., 105, 331-337.

27) Libby, P. (2003) Vascular biology of atherosclerosis: Overview and state of art. Am. J. Cardiol., 91(suppl), 3A-6A.

28) Jacobson, M. D. (1996) Reactive oxygen species and programmed cell death. Trends Biochem. Sci., 243, 81-119.

29) Tomoda, H., Morimoto, K. and Aoki, N. (1996) Superoxide dismutase activity as a predictor of myocardial reperfusion and salvage in acute myocardial infarction. Am. Heart J., 131, 849-856.

30) Dubois-Rande, J. L., Artigou, J., Darmon, J. Y., Habbal, R., Manuel, C., Tayarani, I., and Castaigne, A. (1994) Oxidative stress in patients with unstable angina. Eur. Heart J., 15, 179-183.

31) McMurray, J. (1993) Evidence of oxidative stress in chronic heart failure in humans. Eur. Heart J., 14, 1493-1497.

32) Verma, V. K., Ramesh, V., Tewari, S., Gupta, R. K., Sinha, N. and Pandey, C. M. (2005) Role of Bilirubin, Vitamin $\mathrm{C}$ and Ceruloplasmin as antioxidants in Coronary Artery Disease (CAD). Indian J. Clin. Biochem., 20, 68-74.

33) Kharb, S. (2003) Low blood glutathione levels in acute myocardial infarction. Indian J. Med. Sci., 57, 335-337.

34) Bar-Or, D., Lau, E., Rao, N., Bampos, N., Winkler, J. V. and Curtis, C. G. (1999) Reduction in the cobalt binding capacity of human albumin with myocardial ischemia. Ann. Emerg. Med., 34, 556. 